\title{
ON ESTIMATION OF THE SHAPE PARAMETER OF THE GAMMA DISTRIBUTION
}

\author{
O. Zaihraiev, A. Podraza-Karakulska \\ Nicolaus Copernicus University \\ Toruń, POLAND \\ e-mail: alzaig@mat.uni.torun.pl
}

\begin{abstract}
The problem of estimation of an unknown shape parameter under the sample drawn from the gamma distribution, where the scale parameter is also unknown, is considered. A new estimator, called the maximum likelihood scale invariant estimator, is proposed. It is established that the mean square error of this estimator is less than that of the usual maximum likelihood estimator. The asymptotics of the mean square error of the new estimator is also obtained.
\end{abstract}

\section{Introduction}

Let a sample $x=\left(x_{1}, x_{2}, \ldots, x_{n}\right)$ be drawn from the gamma distribution $\Gamma(\alpha, \sigma)$ with an unknown shape parameter $\alpha>0$ and an unknown scale parameter $\sigma>0$, whose density function has the form

$$
p(u ; \alpha, \sigma)=\frac{u^{\alpha-1} e^{-u / \sigma}}{\sigma^{\alpha} \Gamma(\alpha)}, \quad u>0 .
$$

Consider the problem of estimation of $\alpha$. One of the most popular estimator is the well-known maximum likelihood estimator (ML-estimator) (e.g. [2, Sections 9.3, 9.4], [3], [4], [5]). Let

$$
\mathbf{p}(x ; \alpha, \sigma)=\sigma^{-n \alpha}(\Gamma(\alpha))^{-n}\left(\prod_{j=1}^{n} x_{j}\right)^{\alpha-1} \exp \left(-\sum_{k=1}^{n} x_{k} / \sigma\right)
$$

be the corresponding likelihood function. The ML-estimators of $\alpha$ and $\sigma$ are determined by the equations:

$$
\left\{\begin{array}{l}
\ln \sigma+\Psi(\alpha)=\sum_{j=1}^{n} \ln x_{j} / n, \\
\alpha-\sum_{k=1}^{n} x_{k} /(n \sigma)=0,
\end{array}\right.
$$

where $\Psi(\alpha)=(\ln \Gamma(\alpha))^{\prime}$ is the so-called Euler psi (digamma) function.

From those equations one can obtain the ML-estimators $\alpha^{*}$ and $\sigma^{*}$. Namely, $\alpha^{*}$ is the root of the equation $g(\alpha)=T(x)$, where

$$
g(\alpha)=\ln \alpha-\Psi(\alpha), \quad T(x)=\ln \bar{x}-\frac{1}{n} \sum_{j=1}^{n} \ln x_{j}
$$


while

$$
\sigma^{*}=\frac{\bar{x}}{\alpha^{*}}
$$

Here, $\bar{x}$ is the sample mean, i.e.

$$
\bar{x}=\frac{1}{n} \sum_{k=1}^{n} x_{k} .
$$

Observe that the function $g$ is strictly decreasing and takes values in $(0, \infty)$ (e.g. Theorem 1 of [1]). Therefore, the estimator $\alpha^{*}$ is well-defined and unique. This estimator is also scale invariant. Furthermore, one can easily see that $E T(x)=g_{n}(\alpha)$, where

$$
g_{n}(\alpha)=g(\alpha)-g(n \alpha)=\Psi(n \alpha)-\Psi(\alpha)-\ln n .
$$

The question arises: why don't one takes the root of the equation

$$
g_{n}(\alpha)=T(x)
$$

as an estimator of an unknown shape parameter $\alpha$ ?

It turns out that such a choice has quite a deep reasoning. Since in our scheme $\sigma$ becomes a nuisance parameter, it is natural to apply the maximum likelihood principle to the measure defined on the $\sigma$-algebra of the scale invariant sets generated by the underlying gamma distribution. As it is known (e.g. [6, Section 8.3]), the density corresponding to this measure, with respect to that generated by the standard normal distribution, is given as follows:

$$
\mathbf{q}(x ; \alpha)=\frac{\int_{0}^{\infty} t^{n-1} \mathbf{p}(t x ; \alpha, \sigma) d t}{\int_{0}^{\infty} t^{n-1} \mathbf{s}(t x) d t}=\frac{2 \pi^{n / 2} \Gamma(n \alpha)\left(\sum_{i=1}^{n} x_{i}^{2}\right)^{n / 2}\left(\prod_{i=1}^{n} x_{i}\right)^{\alpha-1}}{\Gamma(n / 2)(\Gamma(\alpha))^{n}\left(\sum_{i=1}^{n} x_{i}\right)^{n \alpha}},
$$

where

$$
\mathbf{s}(u)=(2 \pi)^{-n / 2} \exp \left(-\frac{1}{2} \sum_{k=1}^{n} u_{k}^{2}\right) .
$$

Then by direct calculations one can obtain that the maximum likelihood scale invariant estimator (IML-estimator) $\alpha^{* *} \in \arg \max _{\alpha>0} \mathbf{q}(x ; \alpha)$ is the root of equation (1).

Of course, the estimator $\alpha^{* *}$ is scale invariant, well-defined and unique since the function $g_{n}$ is strictly decreasing and takes values in $(0, \infty)$.

The goal of this paper is to compare two estimators of $\alpha$ : the ML-estimator and the IML-estimator. Taking as a measure of the estimator quality it's mean square error, we show that the IML-estimator $\alpha^{* *}$ is better than the ML-estimator $\alpha^{*}$.

\section{Main results}

Denote

$$
R_{n}^{*}=E\left(\alpha^{*}-\alpha\right)^{2}, \quad R_{n}^{* *}=E\left(\alpha^{* *}-\alpha\right)^{2} .
$$

Theorem 1. If a sample $x=\left(x_{1}, x_{2}, \ldots, x_{n}\right)$ is drawn from $\Gamma(\alpha, \sigma)$ distribution, then the IML-estimator $\alpha^{* *}$ is better than the $M L$-estimator $\alpha^{*}$, i.e. $R_{n}^{* *}<R_{n}^{*}$. 
Remark. From the proof of Theorem 1 it follows that $\alpha^{*}>\alpha^{* *}$ for any sample $x$ and that $E \alpha^{* *}>\alpha$ for any $\alpha>0$. We apply the Monte-Carlo simulation to confirm those facts. Given $\alpha$ and $\sigma$, we generate 10000 samples drawn from $\Gamma(\alpha, \sigma)$ distribution for $n=10,20,30,50,100$. Next, we solve numerically two equations: $g(\alpha)=T(x)$ and $g_{n}(\alpha)=T(x)$ with respect to $\alpha$, and obtain 10000 values of $\alpha^{*}$ and $\alpha^{* *}$. Taking their means, we get $E \alpha^{*}$ and $E \alpha^{* *}$ and calculate $R_{n}^{*}$ and $R_{n}^{* *}$. The results are given in Table 1.

\begin{tabular}{|c|c|c|c|c|c|c|c|c|}
\hline \multicolumn{5}{|c|}{$\alpha=0.125$} & \multicolumn{4}{|c|}{$\alpha=0.5$} \\
\hline$n$ & $E \alpha^{*}$ & $E \alpha^{* *}$ & $R_{n}^{*}$ & $R_{n}^{* *}$ & $E \alpha^{*}$ & $E \alpha^{* *}$ & $R_{n}^{*}$ & $R_{n}^{* *}$ \\
\hline 10 & 0.1533 & 0.1435 & 0.0048 & 0.0038 & 0.5571 & 0.5325 & 0.0320 & 0.0308 \\
\hline 20 & 0.1372 & 0.1331 & 0.0014 & 0.0013 & 0.5473 & 0.5303 & 0.0219 & 0.0198 \\
\hline 30 & 0.1330 & 0.1304 & 0.00082 & 0.00076 & 0.5367 & 0.5248 & 0.0152 & 0.0139 \\
\hline 50 & 0.1298 & 0.1283 & 0.00042 & 0.00040 & 0.5225 & 0.5153 & 0.0089 & 0.0083 \\
\hline 100 & 0.1272 & 0.1265 & 0.00019 & 0.00018 & 0.5108 & 0.5073 & 0.0038 & 0.0037 \\
\hline \multicolumn{5}{|c|}{$\alpha=1$} & \multicolumn{4}{|c|}{$\alpha=2$} \\
\hline$n$ & $\overline{E \alpha^{*}}$ & $E \alpha^{* *}$ & $\overline{R_{n}^{*}}$ & $R_{n}^{* *}$ & $E \alpha^{*}$ & $E \alpha^{* *}$ & $R_{n}^{*}$ & $R_{n}^{* *}$ \\
\hline 10 & 1.1228 & 1.0733 & 0.1324 & 0.1261 & 2.2330 & 2.1222 & 0.5351 & 0.5024 \\
\hline 20 & 1.1030 & 1.0667 & 0.0977 & 0.0892 & 2.2169 & 2.1406 & 0.4159 & 0.3824 \\
\hline 30 & 1.0807 & 1.0543 & 0.0701 & 0.0644 & 2.1718 & 2.1141 & 0.3122 & 0.2861 \\
\hline 50 & 1.0505 & 1.0342 & 0.0395 & 0.0369 & 2.1179 & 2.0817 & 0.1866 & 0.1738 \\
\hline 100 & 1.0277 & 1.0197 & 0.0182 & 0.0175 & 2.0511 & 2.0333 & 0.0795 & 0.0765 \\
\hline \multicolumn{5}{|c|}{$\alpha=4$} & \multicolumn{4}{|c|}{$\alpha=8$} \\
\hline$n$ & $\overline{E \alpha^{*}}$ & $E \alpha^{* *}$ & $\overline{R_{n}^{*}}$ & $\overline{R_{n}^{* *}}$ & $E \alpha^{*}$ & $E \alpha^{* *}$ & $\overline{R_{n}^{*}}$ & $R_{n}^{* *}$ \\
\hline 10 & 4.4238 & 4.1880 & 2.1022 & 1.9780 & 8.9147 & 8.4528 & 8.7071 & 8.2858 \\
\hline 20 & 4.4220 & 4.2628 & 1.7076 & 1.5750 & 8.8514 & 8.5149 & 6.9795 & 6.3896 \\
\hline 30 & 4.3624 & 4.2398 & 1.2980 & 1.1853 & 8.7067 & 8.4591 & 5.3588 & 4.9447 \\
\hline 50 & 4.2595 & 4.1809 & 0.7973 & 0.7353 & 8.4543 & 8.2935 & 3.1421 & 2.9171 \\
\hline 100 & 4.1121 & 4.0740 & 0.3375 & 0.3240 & 8.2298 & 8.1507 & 1.4218 & 1.3644 \\
\hline \multicolumn{5}{|c|}{$\alpha=16$} & \multicolumn{4}{|c|}{$\alpha=32$} \\
\hline $\mathrm{n}$ & $E \alpha^{*}$ & $E \bar{E}^{* *}$ & $\overline{R_{n}^{*}}$ & $\overline{R_{n}^{* *}}$ & $E \alpha^{*}$ & $\bar{E} E \alpha^{* *}$ & 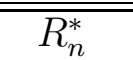 & 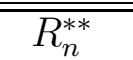 \\
\hline 10 & 17.78 & 16.92 & 34.35 & 33.87 & 35.51 & 33.60 & 138.39 & 131.67 \\
\hline 20 & 17.79 & 17.11 & 28.88 & 26.44 & 35.50 & 34.18 & 115.15 & 106.82 \\
\hline 30 & 17.52 & 16.99 & 21.99 & 19.83 & 34.91 & 33.88 & 86.39 & 79.42 \\
\hline 50 & 17.01 & 16.69 & 13.30 & 12.47 & 33.95 & 33.29 & 52.90 & 49.18 \\
\hline 100 & 16.49 & 16.36 & 5.80 & 5.56 & 33.03 & 32.71 & 24.31 & 23.28 \\
\hline
\end{tabular}

Table 1: Numerical calculations of $E \alpha^{*}, E \alpha^{* *}, R_{n}^{*}, R_{n}^{* *}$.

In the next theorem we establish the asymptotics of the mean square error of the maximum likelihood scale invariant estimator with respect to that of the usual maximum likelihood estimator. 
Theorem 2. If a sample $x=\left(x_{1}, x_{2}, \ldots, x_{n}\right)$ is drawn from $\Gamma(\alpha, \sigma)$ distribution, then

$$
n^{2}\left(R_{n}^{*}-R_{n}^{* *}\right)=\frac{3}{4} D(\alpha)+o(1), \quad n \rightarrow \infty
$$

where

$$
D(\alpha)=-\frac{1+\alpha \Psi^{\prime}(\alpha)+2 \alpha^{2} \Psi^{\prime \prime}(\alpha)}{\left(\alpha \Psi^{\prime}(\alpha)-1\right)^{3}}>0
$$

\section{References}

[1] Alzer H. (1997). On some inequalities for the gamma and psi functions. Math. Comp. Vol. 66, pp. 373-389.

[2] Barndorff-Nielsen O. (1978). Information and Exponential families in Statistical Theory. Wiley, New York.

[3] Bowman K.O., Shenton L.R. (1988). Properties of Estimators for the Gamma Distribution. Marcel Dekker, New York.

[4] Dang H., Weerakkody G. (2000). Bounds for the maximum likelihood estimates in two-parameter gamma distribution. J. Math. Anal. Appl. Vol. 245, pp. 1-6.

[5] Crain B.R. (1976). Exponential models, maximum likelihood estimation, and the Haar condition. J. Amer. Statist. Assoc. Vol. 71, pp. 737-740.

[6] Nagaev A.V. (1996). Limit Theorems under Testing Hypotheses. UMK, Torun. 